\title{
Fault Diagnosis of Bearing Based on Cauchy Kernel Relevance Vector Machine Classifier with SIWPSO
}

\author{
Sheng-wei Fei and Yong He \\ School of Mechanical Engineering, Donghua University, Shanghai 201620, China \\ Correspondence should be addressed to Sheng-wei Fei; fei1980s@163.com
}

Received 21 January 2015; Revised 15 May 2015; Accepted 20 May 2015

Academic Editor: Laurent Mevel

Copyright (C) 2015 S.-w. Fei and Y. He. This is an open access article distributed under the Creative Commons Attribution License, which permits unrestricted use, distribution, and reproduction in any medium, provided the original work is properly cited.

Bearing is an important component of mechanical system; any defects of bearing will lead to serious damage for the entire mechanical system. In this paper, Cauchy kernel relevance vector machine with stochastic inertia weight particle swarm optimization algorithm (SIWPSO-CauchyRVM) is proposed to fault diagnosis for bearing. As the selection of the Cauchy kernel parameter has a certain influence on the diagnosis result of relevance vector machine, stochastic inertia weight PSO is used to select the Cauchy kernel parameter. The relative energies of 16 wavelet coefficients of the forth layer of vibration signal of bearing can be used as the diagnosis features of bearing. The experimental results indicate that fault diagnosis method of bearing based on SIWPSO-CauchyRVM has excellent diagnosis ability.

\section{Introduction}

Bearing is an important component of mechanical system; any defects of bearing will lead to serious damage for the entire mechanical system $[1,2]$. Therefore, it is essential to develop the reliable fault diagnosis method to prevent the bearing from malfunction [3-5]. Vibration signal analysis is the most common method for detecting bearing failures [68], and, on the basis of vibration signal analysis, intelligent fault diagnosis methods have been applied in fault diagnosis for bearing. Support vector machine (SVM) is a machine learning method based on structure risk minimization principle, which has been applied to fault diagnosis for bearing in the past few years $[9,10]$ due to its good nonlinear classification ability. Relevance vector machine (RVM) is an intelligent learning technique based on sparse Bayesian framework [11]. The number of relevance vectors in RVM is much smaller than that of support vectors in SVM, which makes RVM have a sparser representation compared with SVM. In addition, there is no need to set the penalty parameter in RVM, which makes RVM more convenient to use than SVM. Thus, RVM has a better application prospect in fault diagnosis for bearing.

In this paper, Cauchy kernel relevance vector machine with stochastic inertia weight particle swarm optimization algorithm (SIWPSO-CauchyRVM) is proposed to fault diagnosis for bearing. As the selection of the Cauchy kernel parameter has a certain influence on the diagnosis result of relevance vector machine, stochastic inertia weight PSO is used to select the Cauchy kernel parameter. As the relative energies of 16 wavelet coefficients of the forth layer of vibration signal of bearing have a good discrimination for different faults of bearing, it can be used as the diagnosis features of bearing. As multiclass classification model can be established by combining multi-RVM binary classifiers, three SIWPSOCauchyRVMs are employed to establish the diagnosis model with the form of binary tree. The experimental results indicate that fault diagnosis method of bearing based on SIWPSOCauchyRVM has excellent diagnosis ability.

\section{Cauchy Kernel RVM Classifier}

Relevance vector machine is a machine learning method with Bayesian framework, and multiclass classification model can be established by combining multi-RVM binary classifiers.

Given a set of the training samples $\mathbf{D}=(\mathbf{X}, \mathbf{T}), \mathbf{X}=$ $\left\{\mathbf{x}_{n}\right\}_{n=1}^{N}, \mathbf{T}=\left\{t_{n}\right\}_{n=1}^{N}, t_{n} \in\{0,1\}$, “0" and "1" denote two classes which the training samples belong to and $N$ denotes 


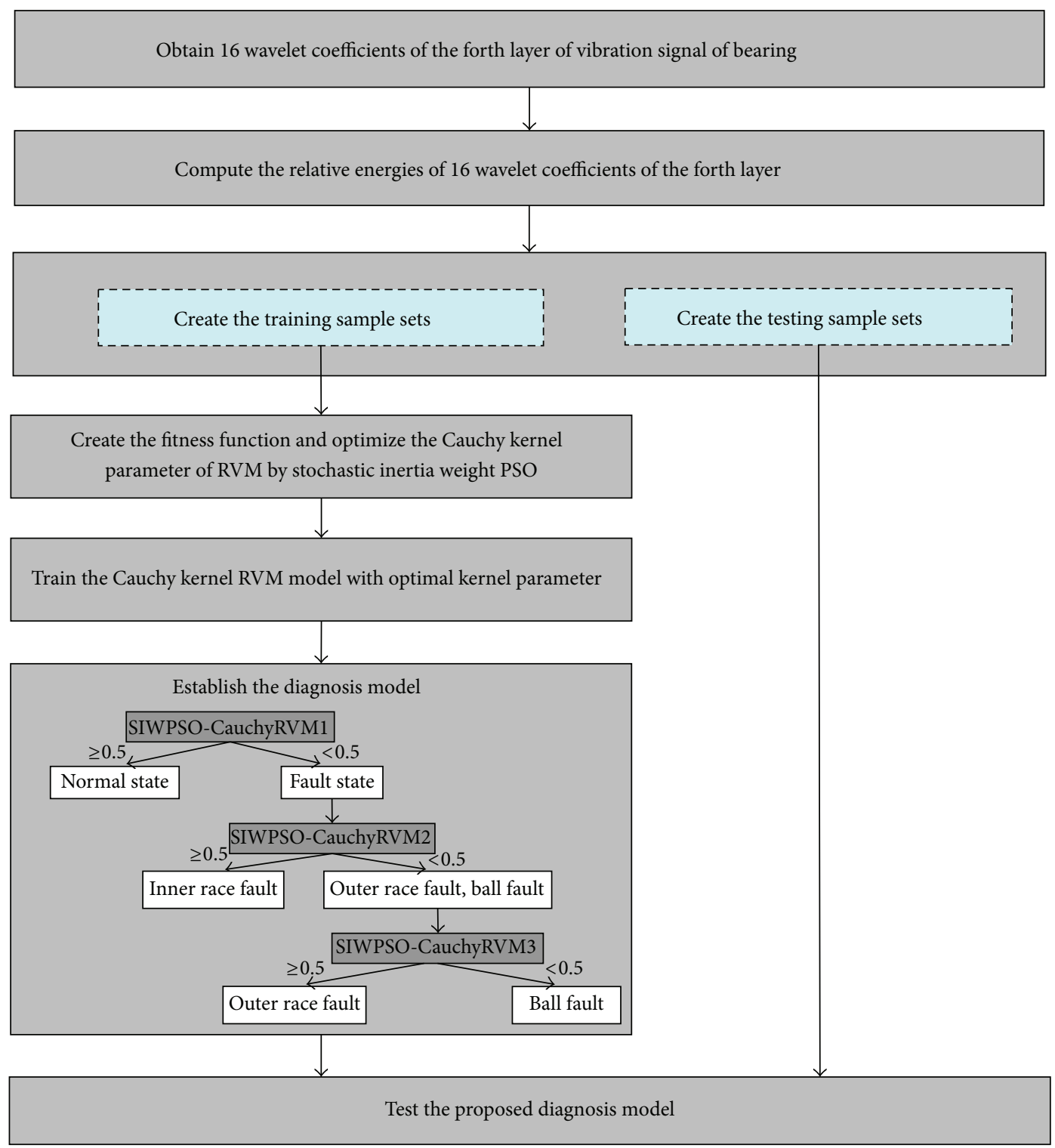

FIGURE 1: Fault diagnosis process of bearing based on Cauchy kernel relevance vector machine with stochastic inertia weight PSO.

the number of the training samples. The likelihood function obeys the Bernoulli distribution [11]:

$$
P(\mathbf{T} \mid \mathbf{X})=\prod_{n=1}^{N} \sigma\left\{y\left(\mathbf{x}_{n} ; \mathbf{W}\right)\right\}^{t_{n}}\left[1-\sigma\left\{y\left(\mathbf{x}_{n} ; \mathbf{W}\right)\right\}\right]^{1-t_{n}}
$$

where $\sigma\left(y\left(\mathbf{x}_{n} ; \mathbf{W}\right)\right)$ is a predefined logistic sigmoid function, $\sigma\left(y\left(\mathbf{x}_{n} ; \mathbf{W}\right)\right)=1 /\left(1+e^{-y\left(\mathbf{x}_{n} ; \mathbf{W}\right)}\right)$, and $\mathbf{W}$ is the weight vector.

The detailed description of RVM binary classifier can be shown in [11]. Cauchy kernel function is employed in this RVM, which can be expressed as follows:

$$
K_{\text {Cauchy }}\left(\mathbf{x}_{n}, \mathbf{x}_{i}\right)=\frac{1}{1+\left\|\mathbf{x}_{n}-\mathbf{x}_{i}\right\|^{2} / q^{2}},
$$

where $q$ is the Cauchy kernel parameter.

\section{Fault Diagnosis Process of Bearing Based on Cauchy Kernel Relevance Vector Machine with Stochastic Inertia Weight PSO}

Figure 1 shows the fault diagnosis process of bearing based on Cauchy kernel relevance vector machine with stochastic inertia weight PSO; first, we perform 4-layer wavelet decomposition for vibration signal of bearing, and obtain 16 wavelet coefficients of the forth layer of vibration signal of bearing and then compute the relative energies of 16 wavelet coefficients of the forth layer, which can be used as the diagnosis features of bearing.

As the selection of the Cauchy kernel parameter has a certain influence on the diagnosis result of relevance vector machine, stochastic inertia weight PSO is used to select the Cauchy kernel parameter $q$; the fitting diagnosis error of 

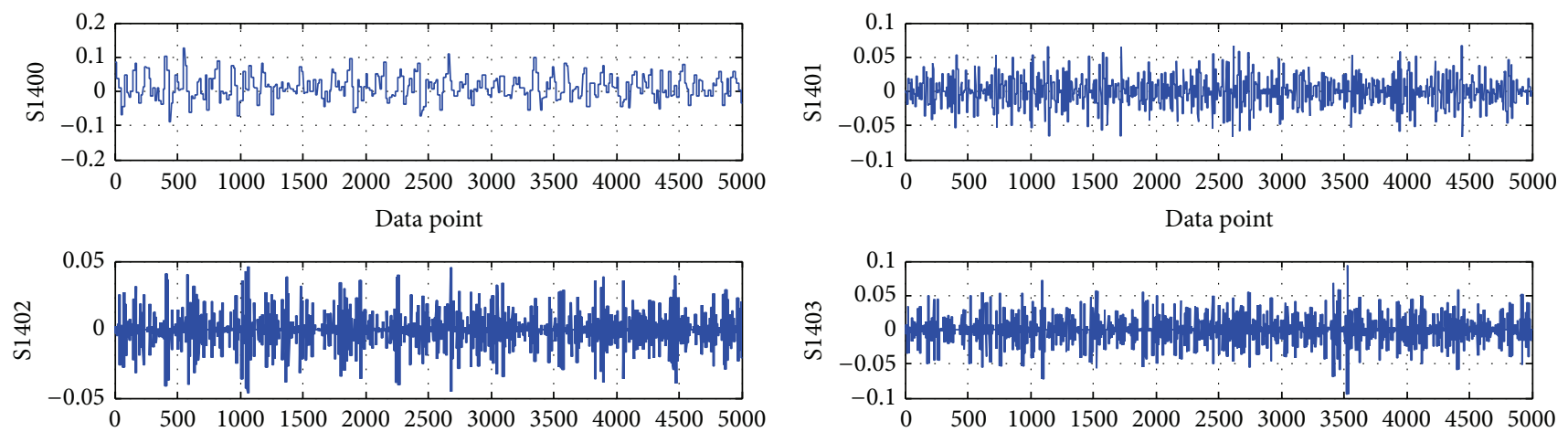

Data point
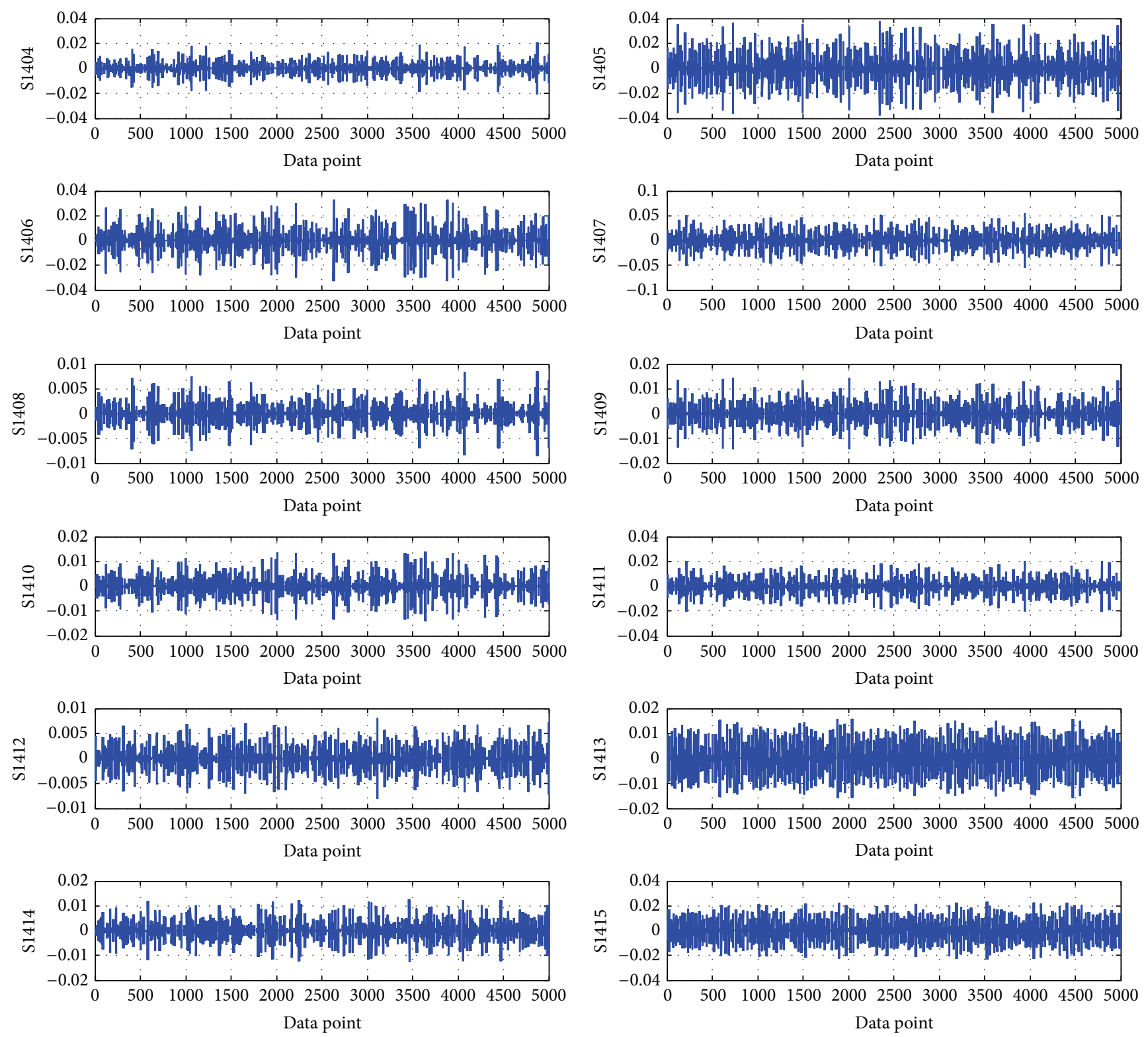

FIGURE 2: 16 wavelet coefficients of one of the samples representing normal state. 

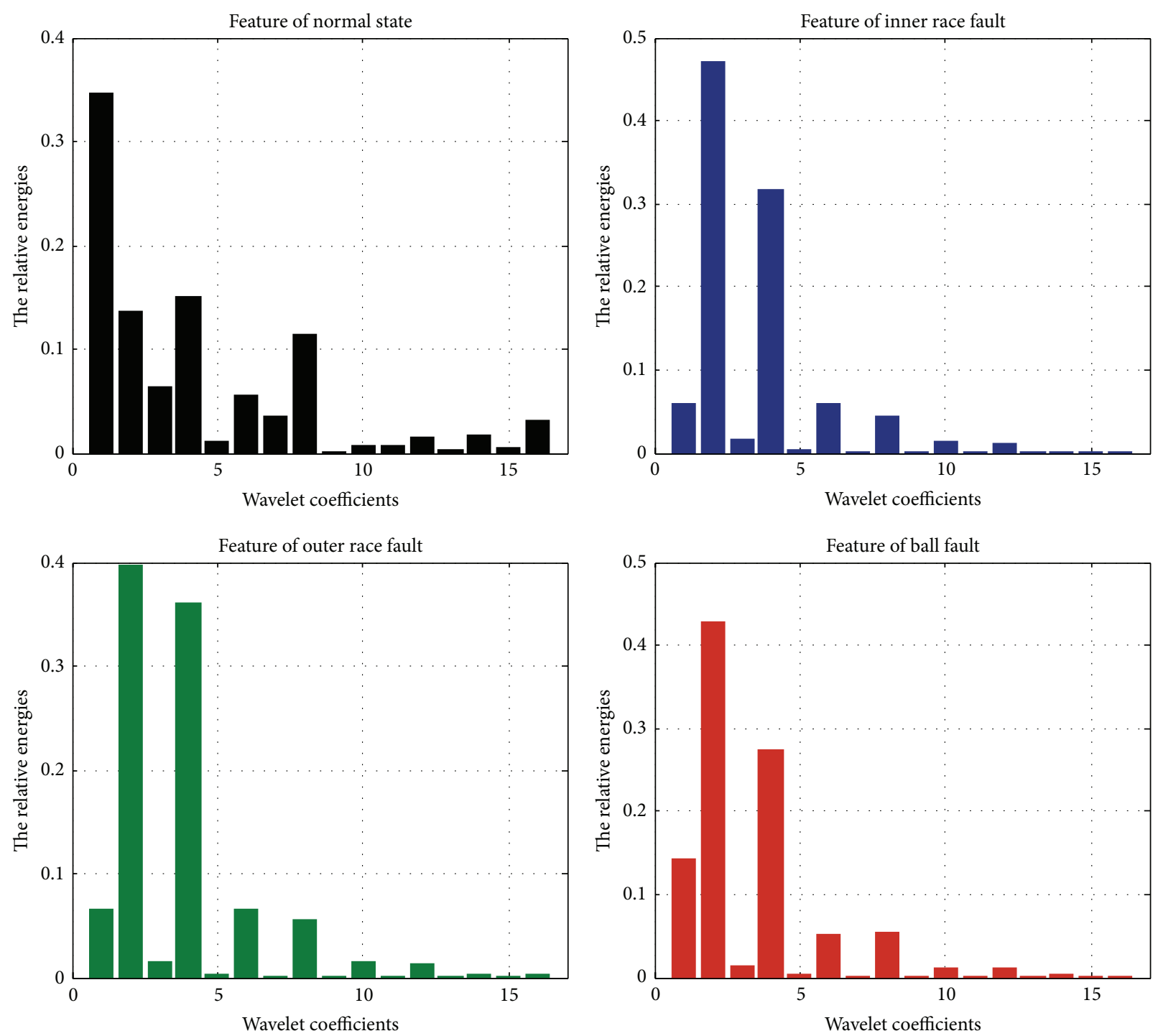

FIGURE 3: The features of a group of samples with different states.

all the training samples is used to evaluate the fitness of the current Cauchy kernel parameter $q$. The process of the selection of the Cauchy kernel parameter $q$ of relevance vector machine by stochastic inertia weight PSO can be described as follows.

Step 1. Perform the setting of the parameters of SIWPSO and initialization of the particles.

Step 2. The fitting diagnosis error for all the training samples is used to create the fitness function and evaluate the fitness of each current particle according to the following formula:

$$
\widetilde{e}_{k}=1-\frac{S_{k}}{H_{k}}
$$

where $S_{k}$ is the number of the samples of correct fitting diagnosis for the training samples of the $k$ th RVM and $H_{k}$ is the number of the training samples of the $k$ th RVM.
Step 3. Update the global and personal best according to the fitness evaluation results.

Step 4 . The particle flies toward a new position by the velocity is calculated by the following formula [12]:

$$
\begin{aligned}
v_{i j}(t+1)= & \omega \cdot v_{i j}(t)+c_{1} \cdot \text { rand } \cdot\left(\text { Pbest }_{i j}(t)-p_{i j}(t)\right) \\
& +c_{2} \cdot \text { rand } \cdot\left(\text { Gbest }_{j}(t)-p_{i j}(t)\right),
\end{aligned}
$$

where $t$ denotes the iteration counter and rand denotes the random value in the range $[0,1] ; v_{i j}$ is the velocity of particle $i$ on the $j$ th dimension and $p_{i j}$ is the position of particle $i$ on the $j$ th dimension; Pbest $_{i j}$ is the personal best position of particle $i$ on the $j$ th dimension and Gbest ${ }_{j}$ is the global best position of the swarm on the $j$ th dimension; $c_{1}$ and $c_{2}$ are personal and social learning factors, respectively, and $\omega$ is the inertia weight, which is used to balance the global exploration and local exploitation. 


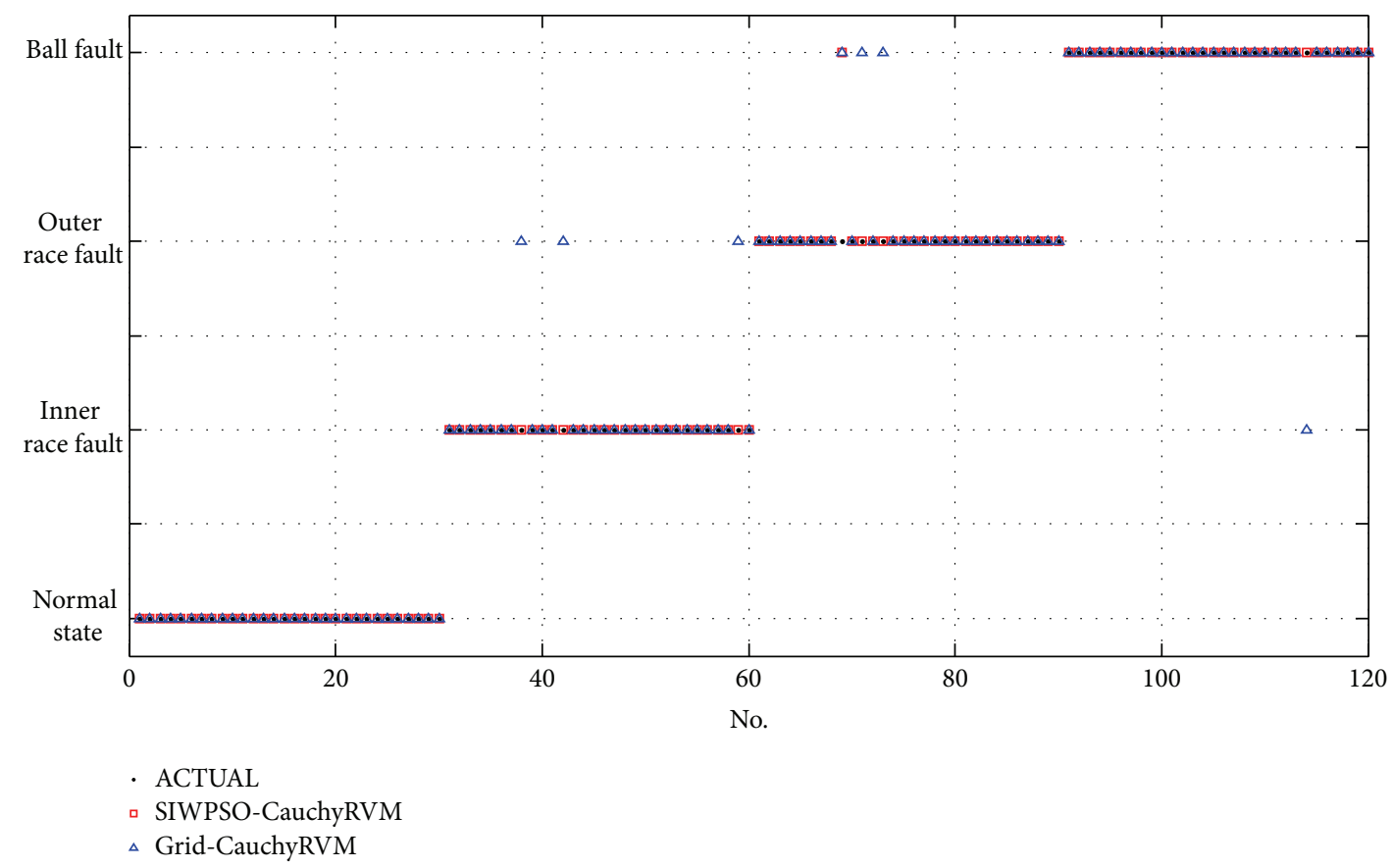

FIGURE 4: The comparison of the diagnosis results between SIWPSO-CauchyRVM and Grid-CauchyRVM in experiment 1.

In stochastic inertia weight PSO, stochastic inertia weight is used, which can be expressed as follows:

$$
\omega=\mu_{\min }+\operatorname{rand} \cdot\left(\mu_{\max }-\mu_{\min }\right)+\delta \cdot N(0,1)
$$

where $N(0,1)$ denotes a random number from the standard normal distribution; $\delta$ denotes the variance of stochastic inertia weight; $\mu_{\max }$ denotes maximum average inertia weight; and $\mu_{\min }$ denotes minimum average inertia weight.

Step 5. Each particle moves to its next position according to the following formula:

$$
p_{i j}(t+1)=p_{i j}(t)+\beta \cdot v_{i j}(t+1),
$$

where $\beta$ is constraint factor used to control the velocity weight.

Step 6. The same procedures from Step 2 to Step 5 are repeated until the stop conditions are satisfied.

Then, the Cauchy kernel RVM model is trained with the suitable kernel parameter. As shown in Figure 1, three SIWPSO-CauchyRVMs are employed to establish the diagnosis model with the form of binary tree, among which SIWPSO-CauchyRVM1 is used to separate normal state from fault state, SIWPSO-CauchyRVM2 is used to separate inner race fault from other faults, and SIWPSO-CauchyRVM3 is used to separate outer race fault from ball fault. Finally, the proposed SIWPSO-CauchyRVM model can be tested by the testing data.

\section{Experimental Analysis}

In this study, 360 samples including 90 samples representing normal state, 90 samples representing inner race fault, 90 samples representing outer race fault, and 90 samples representing ball fault are used as the experimental data. 16 wavelet coefficients of one of the samples representing normal state can be shown in Figure 2. The relative energies of 16 wavelet coefficients of the forth layer of vibration signal of bearing can be used as the diagnosis features of bearing and the features of a group of samples with different states are shown in Figure 3.

Three experiments with different training sample sets and testing sample sets are performed to testify the feasibility of the proposed model. In the first experiment (experiment 1), 240 samples including the first 60 samples of each state of bearing are used as the training samples; other 120 samples including the remaining 30 samples of each state of bearing are used as the testing samples. In the second experiment (experiment 2), 200 samples including the first 50 samples of each state of bearing are used as the training samples; other 160 samples including the remaining 40 samples of each state of bearing are used as the testing samples. In the third experiment (experiment 3), 160 samples including the first 40 samples of each state of bearing are used as the training samples; other 200 samples including the remaining 50 samples of each state of bearing are used as the testing samples.

Here, stochastic inertia weight PSO is used to select the Cauchy kernel parameter $q$. In SIWPSO, $\mu_{\max }$ is set to 0.8 , $\mu_{\min }$ is set to $0.5, \delta$ is set to $0.2, \beta$ is set to $1 / 1.3$, and the positive constants $c_{1}$ and $c_{2}$ are set to 2. Grid-CauchyRVM is used to compare with stochastic inertia weight PSO-CauchyRVM (SIWPSO-CauchyRVM). In Grid-CauchyRVM, the value 


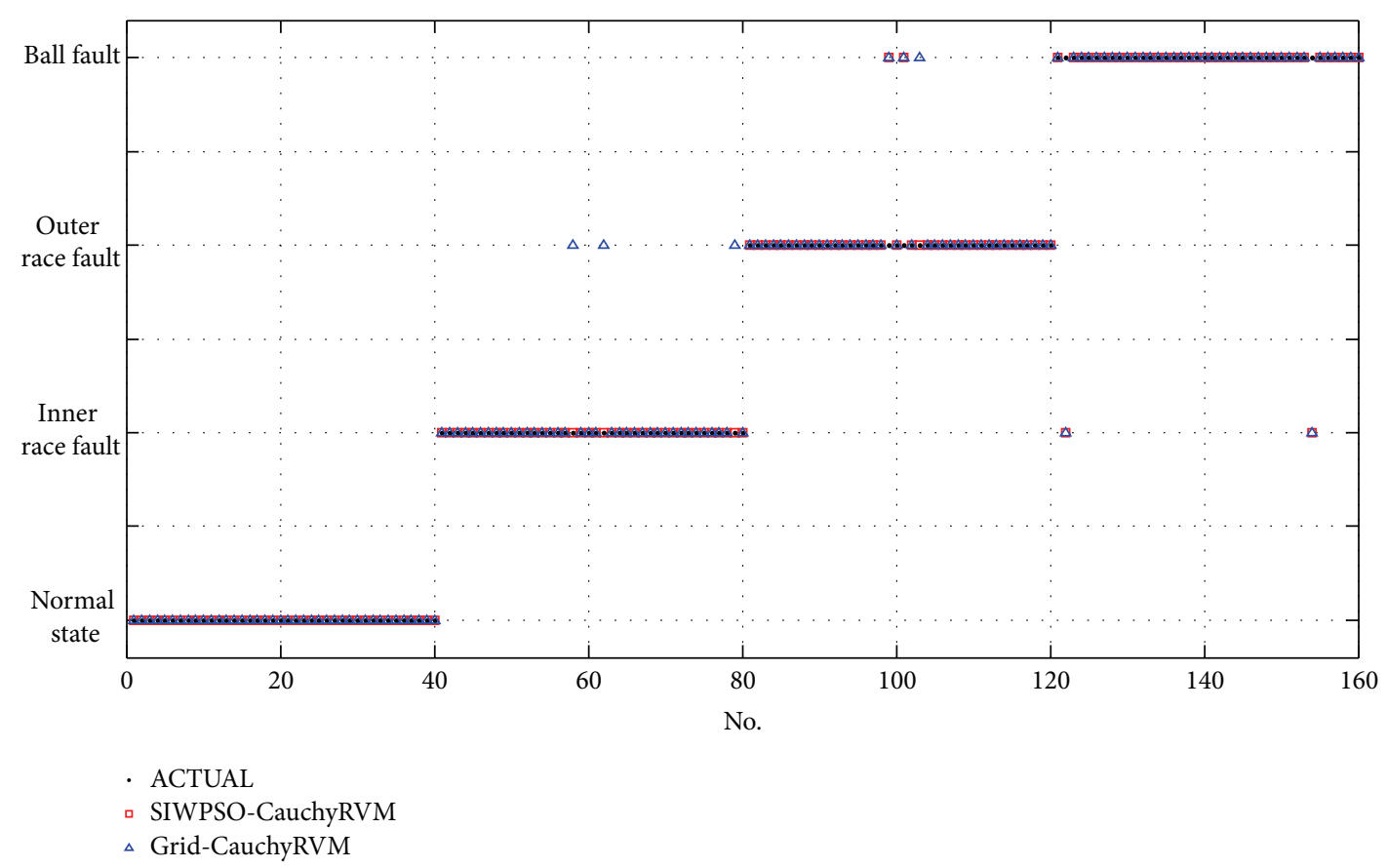

FIGURE 5: The comparison of the diagnosis results between SIWPSO-CauchyRVM and Grid-CauchyRVM in experiment 2.

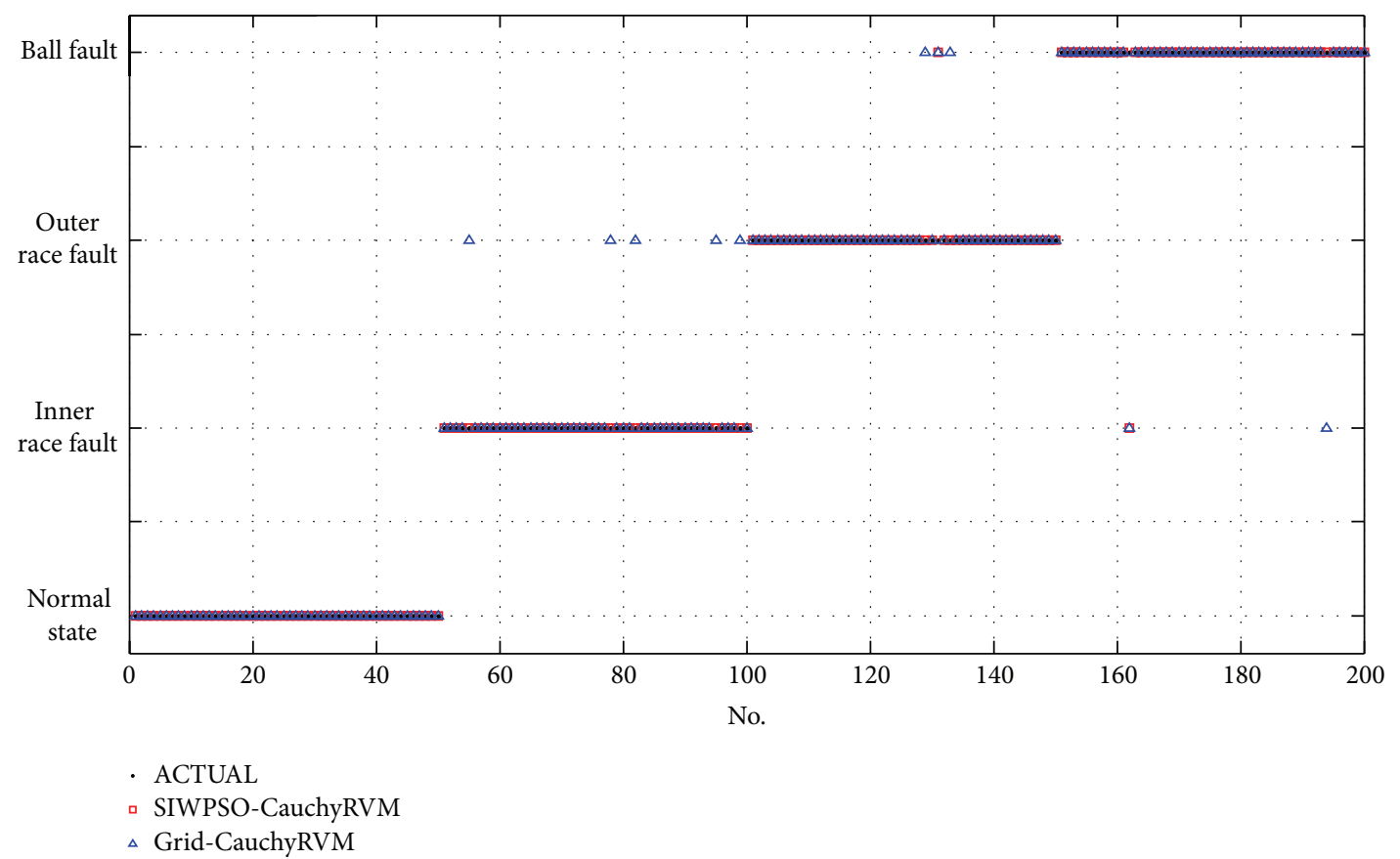

FIGURE 6: The comparison of the diagnosis results between SIWPSO-CauchyRVM and Grid-CauchyRVM in experiment 3.

range of the Cauchy kernel parameter $q$ is between 0 and 2, and the minimum interval of the Cauchy kernel parameter $q$ is 0.01 .

Figure 4 shows the comparison of the diagnosis results between SIWPSO-CauchyRVM and Grid-CauchyRVM in experiment 1, as shown in Table 1, the diagnosis accuracy of SIWPSO-CauchyRVM is $99.167 \%$, and the diagnosis accuracy of Grid-CauchyRVM is $94.167 \%$ in experiment 1. Figure 5 shows the comparison of the diagnosis results between SIWPSO-CauchyRVM and Grid-CauchyRVM in experiment 2, as shown in Table 1, the diagnosis accuracy of SIWPSO-CauchyRVM is $97.5 \%$, and the diagnosis accuracy of Grid-CauchyRVM is $95.0 \%$ in experiment 2. Figure 6 shows the comparison of the diagnosis results between 
TABLE 1: The comparison of the diagnosis accuracies between SIWPSO-CauchyRVM and Grid-CauchyRVM.

\begin{tabular}{lcccc}
\hline & $\begin{array}{c}\text { The number of } \\
\text { training samples }\end{array}$ & $\begin{array}{c}\text { The number of } \\
\text { testing samples }\end{array}$ & $\begin{array}{c}\text { Diagnosis accuracy of } \\
\text { SIWPSO-CauchyRM }\end{array}$ & $\begin{array}{c}\text { Diagnosis accuracy of } \\
\text { Grid-CauchyRVM }\end{array}$ \\
\hline Experiment 1 & 240 & 120 & $99.167 \%$ & $94.167 \%$ \\
Experiment 2 & 200 & 160 & $97.5 \%$ & $95.0 \%$ \\
Experiment 3 & 160 & 200 & $99.0 \%$ & $95.0 \%$ \\
\hline
\end{tabular}

SIWPSO-CauchyRVM and Grid-CauchyRVM in experiment 3 , as shown in Table 1, the diagnosis accuracy of SIWPSOCauchyRVM is $99.0 \%$, and the diagnosis accuracy of GridCauchyRVM is $95.0 \%$ in experiment 3. It can be seen that SIWPSO-CauchyRVM has a higher diagnosis accuracy than Grid-CauchyRVM, and fault diagnosis of bearing based on SIWPSO-CauchyRVM is feasible.

\section{Conclusion}

In this paper, Cauchy kernel relevance vector machine with stochastic inertia weight PSO is proposed to fault diagnosis for bearing, and stochastic inertia weight PSO is used to select the kernel parameter of Cauchy kernel relevance vector machine. The relative energies of 16 wavelet coefficients of the forth layer of vibration signal of bearing can be used as the diagnosis features of bearing, and three SIWPSOCauchyRVMs are employed to establish the diagnosis model with the form of binary tree. The experimental results indicate that SIWPSO-CauchyRVM has a higher diagnosis accuracy than Grid-CauchyRVM, and fault diagnosis of bearing based on SIWPSO-CauchyRVM is feasible.

\section{Conflict of Interests}

The authors declare that they have no conflict of interests.

\section{References}

[1] S. Singh, A. Kumar, and N. Kumar, "Motor current signature analysis for bearing fault detection in mechanical systems," Procedia Materials Science, vol. 6, pp. 171-177, 2014.

[2] D. S. Shah and V. N. Patel, "A review of dynamic modeling and fault identifications methods for rolling element bearing," Procedia Technology, vol. 14, pp. 447-456, 2014.

[3] L. Saidi, J. B. Ali, and F. Fnaiech, "Bi-spectrum based-EMD applied to the non-stationary vibration signals for bearing faults diagnosis," ISA Transactions, vol. 53, no. 5, pp. 1650-1660, 2014.

[4] V. Girondin, K. M. Pekpe, H. Morel, and J.-P. Cassar, "Bearings fault detection in helicopters using frequency readjustment and cyclostationary analysis," Mechanical Systems and Signal Processing, vol. 38, no. 2, pp. 499-514, 2013.

[5] L. Batista, B. Badri, R. Sabourin, and M. Thomas, "A classifier fusion system for bearing fault diagnosis," Expert Systems with Applications, vol. 40, no. 17, pp. 6788-6797, 2013.

[6] M. S. Safizadeh and S. K. Latifi, "Using multi-sensor data fusion for vibration fault diagnosis of rolling element bearings by accelerometer and load cell," Information Fusion, vol. 18, no. 1, pp. 1-8, 2014.
[7] J. Ben Ali, N. Fnaiech, L. Saidi, B. Chebel-Morello, and F. Fnaiech, "Application of empirical mode decomposition and artificial neural network for automatic bearing fault diagnosis based on vibration signals," Applied Acoustics, vol. 89, pp. 16-27, 2015.

[8] J. Zarei, M. A. Tajeddini, and H. R. Karimi, "Vibration analysis for bearing fault detection and classification using an intelligent filter," Mechatronics, vol. 24, no. 2, pp. 151-157, 2014.

[9] C. Rajeswari, B. Sathiyabhama, S. Devendiran, and K. Manivannan, "Bearing fault diagnosis using wavelet packet transform, hybrid PSO and support vector machine," Procedia Engineering, vol. 97, pp. 1772-1783, 2014.

[10] K. C. Gryllias and I. A. Antoniadis, "A Support Vector Machine approach based on physical model training for rolling element bearing fault detection in industrial environments," Engineering Applications of Artificial Intelligence, vol. 25, no. 2, pp. 326-344, 2012.

[11] V. T. Tran, B.-S. Yang, F. Gu, and A. Ball, "Thermal image enhancement using bi-dimensional empirical mode decomposition in combination with relevance vector machine for rotating machinery fault diagnosis," Mechanical Systems and Signal Processing, vol. 38, no. 2, pp. 601-614, 2013.

[12] V. Fathi and G. A. Montazer, "An improvement in RBF learning algorithm based on PSO for real time applications," Neurocomputing, vol. 111, pp. 169-176, 2013. 

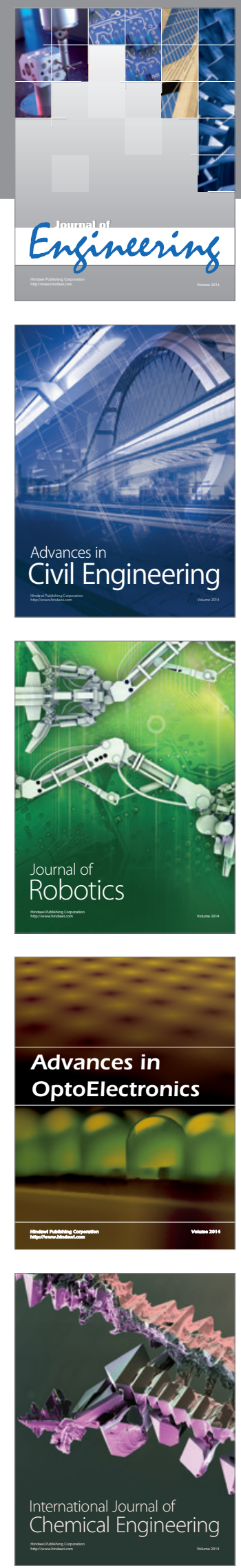

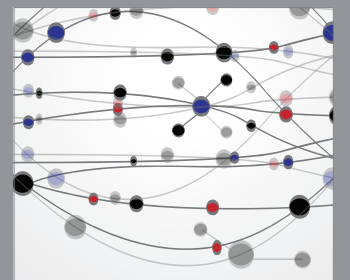

The Scientific World Journal
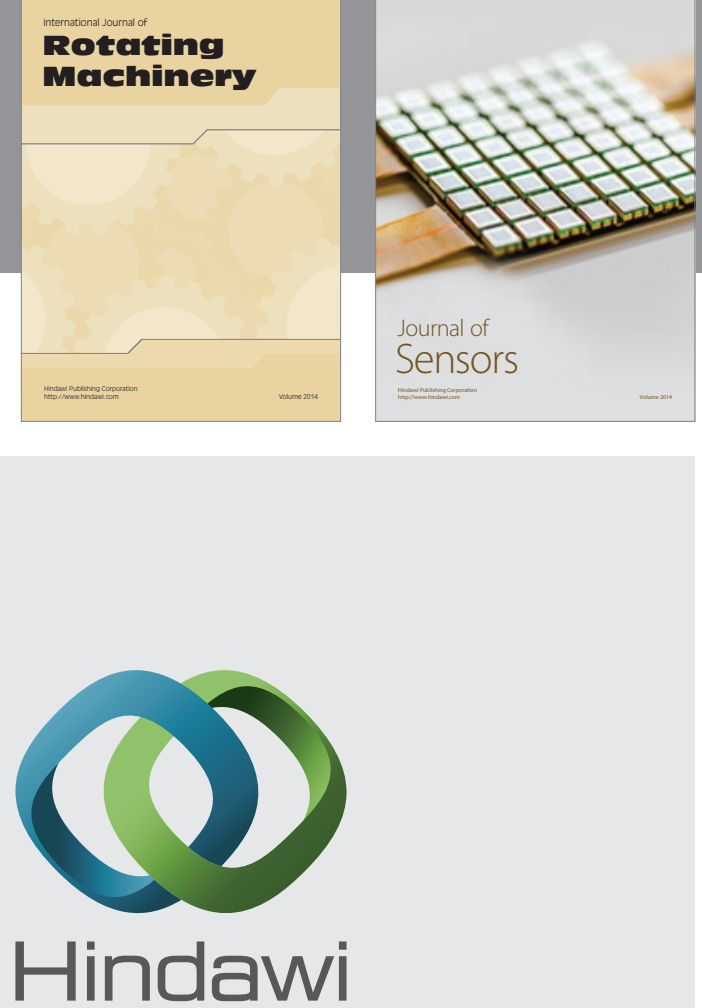

Submit your manuscripts at http://www.hindawi.com
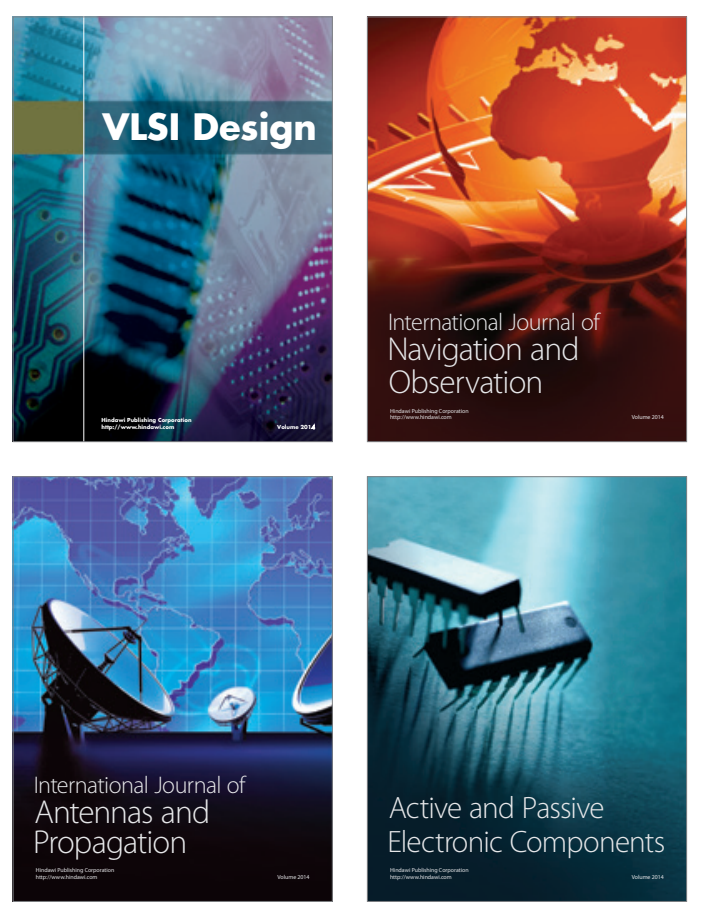
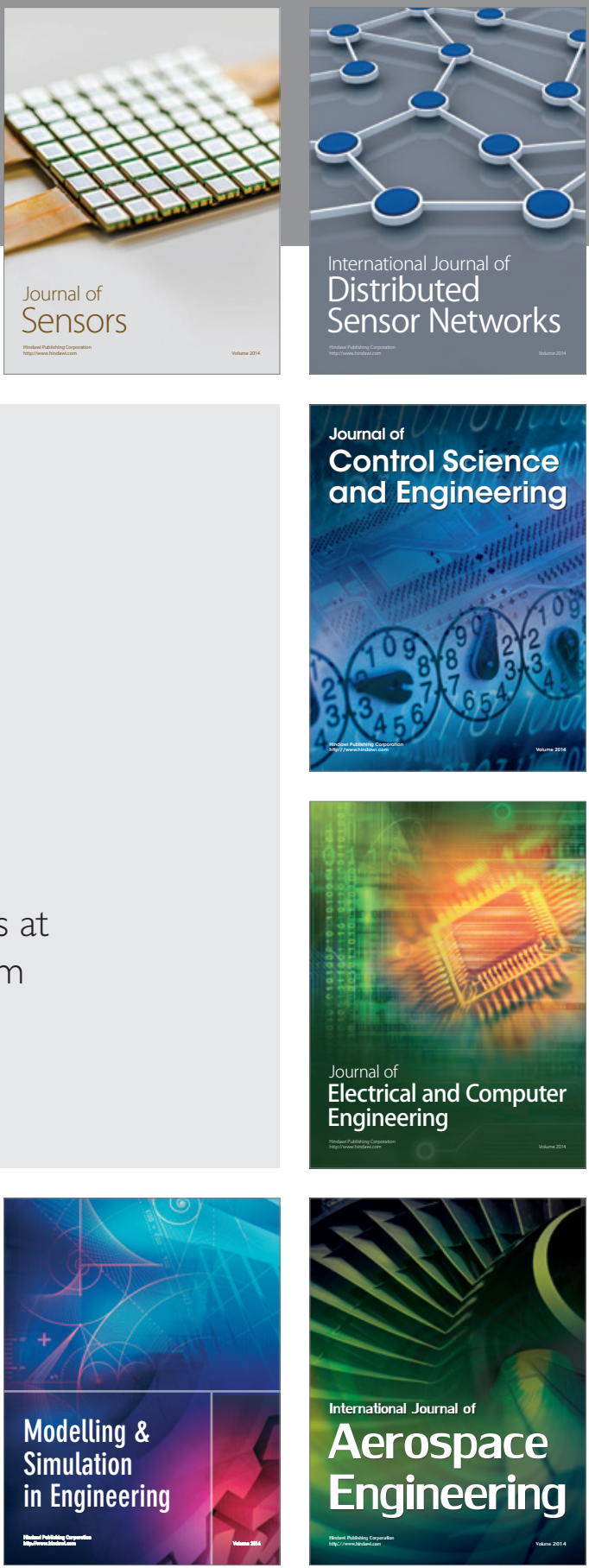

Journal of

Control Science

and Engineering
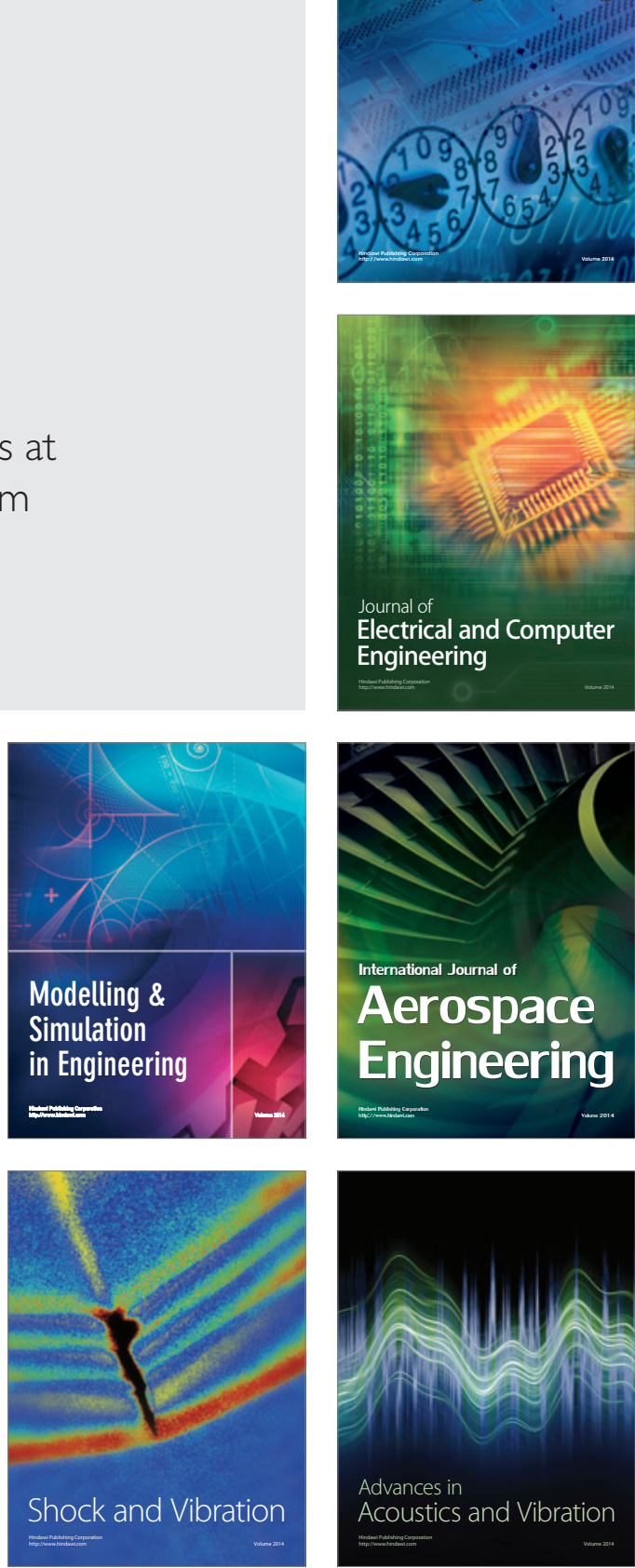